\title{
CURIOUS CASE OF DRUG RESISTANT MALARIA AND ARTEMISININ COMPOUNDS IN THE MODERN ERA
}

\author{
Maqbool $\mathbf{M}^{1}$, Dar $\mathbf{M A}^{1}, \operatorname{Rasool} \mathrm{S}^{1}, \mathrm{Khan}_{\mathbf{M}^{2}}$ \\ ${ }^{1}$ Department of Pharmaceutical Sciences, University of Kashmir, Hazratbal Srinagar-190006, Jammu and Kashmir, India \\ ${ }^{2}$ Mader-E-Meharban Institute of Nursing Sciences and Research, SKIMS, Soura, Jammu and Kashmir, India
}

\section{ABSTRACT}

Malaria is caused by Plasmodium parasite, transmitted by the bite of infective female Anopheles mosquito. The four species causing human infections are $P$ falciparum, $P$ vivax, $P$ malariae and $P$ ovale. $P$ falciparum causes the majority of infections and is main culprit for most severe disease and mortality. Whereas $P$. vivax and $P$. ovale form resting stages in the liver (hypnozoites), that once reactivated, can lead to a clinical relapse many months after the initial event. The effective treatment is now compounded by the spread of drug resistant strains of the parasite. As a result, traditional alkaloid drugs such as chloroquine and quinine are now largely ineffective. The spread of parasite resistance has led the World Health Organization (WHO) to predict that without new antimalarial drug intervention, the number of cases of malaria will definitely increase. The growing menace of drug resistance has greatly complicated the treatment for malaria. Whereas chloroquine and sulfadoxine/pyrimethamine could once cure most infections, this is no longer true and requires examination of alternative regimens for the treatment of malaria. Artemisininbased combinations are now widely accepted as the best treatments for uncomplicated falciparum malaria. Such combination treatments are rapid and reliably effective. The efficacy of the treatment is determined by the drug partnering the artemisinin derivative and, for artesunatemefloquine, artemether-lumefantrine, and dihydroartemisinin-piperaquine, this usually exceeds $95 \%$. This paper unfolds resistance to various conventional antimalarials and brief outline about artemisinin derivatives.

Keywords: Malaria, Chloroquine, Artemisnin, Artesunate, Artemether.

\section{Article info:}

Received: April 18, 2018

Revised: May 20, 2019

Published Online: July, 2019

DOI: https://doi.org/10.31069/japsr.v2i2.1
Correspondence:

Mudasir Maqbool

Department of Pharmaceutical Sciences, University of Kashmir,

Hazratbal Srinagar-190006, Jammu and Kashmir, India.

Phone: +91-9797240145

Email: bhatmudasir.scholar@kashmiruniversity.net

\section{INTRODUCTION}

Anti-malarial drugs can be categorized by the stage of the parasite that they affect and by their intended use for either prophylaxis or treatment. The various stages of malaria life-cycle that occur in humans differ from one another not only in their morphology and metabolism but also in their drug sensitivity. For this reason, the classification of anti-malarial drugs is best done in the context of life-cycle. ${ }^{[1]}$ The effective treatment is now compounded by the spread of drug resistant strains of the parasite. As a result, traditional alkaloid drugs such as chloroquine and quinine are now largely ineffective. ${ }^{[2]}$ The spread of parasite resistance has led the World Health Organization (WHO) to predict that without new anti-malarial drug intervention, the number of cases of malaria will definitely increase. ${ }^{[3]}$ The growing problem of drug resistance has greatly complicated the treatment for falciparum malaria. Whereas chloroquine and sulfadoxine/ pyrimethamine could once cure most infections, this is no longer true and requires examination of alternative regimens. Not all treatment failures are drug resistant and other issues such as expired anti-malarial drugs and patient compliance need to be considered. Continuation of a failing treatment policy after drug resistance is established, suppresses infections rather than curing them, leading to increased transmission of malaria, promotion of epidemics and loss of public confidence in malaria control programs. Over the last generation, falciparum malaria has become widely resistant to a number of common anti-malarial drugs. ${ }^{[4]}{ }^{[5]}$ Drug-resistance arises by the evolutionary selection of spontaneously arising mutants that are drug insensitive. When one kills all sensitive parasites, by definition the ones remaining are drug insensitive. ${ }^{[6]}$ The mechanism of anti-malarial drug-resistance has been investigated at the molecular level and usually it has been found to be due to point mutations that change drug accumulation/efflux in the erythrocyte or reduce drug affinity for the target molecule. Antimalarial drug-resistance may present as a treatment failure in an individual patient, but more commonly it makes its presence known by increasing the apparent number of malaria infections. ${ }^{[7]}$ Individuals appear to be getting multiple infections, when in reality they have a single infection that is suppressed by inadequate treatment followed by a parasite recrudescence. At a community level this is demonstrated by increasing malaria rates, appearance of malaria in previously well-controlled areas and malaria 


\section{Maqbool et al, Malaria and its treatment approaches}

epidemics in marginal transmission zones. Geographic spread of drug-resistance only requires the movement of infected persons into new areas which have competent Anopheles vectors. ${ }^{[8]}$ Maps delineating the geographic extent of multiple drug resistant $P$. falciparum necessarily lag the movement of the parasite and should not be over-interpreted to indicate absence of drugresistance. Multiple drug resistance has certainly arrived in India and is particularly prevalent in the states of the Northeast. Drugresistance is first seen when apparently successfully treated patients recrudesce their infection often weeks after original therapy. Any determination of drug-resistance for public health purposes based on studies with anything less than four weeks of follow-up is bound to be misleading.

Chloroquine-resistance: For nearly a generation after its introduction, Chloroquine successfully cured both falciparum and vivax malaria, greatly simplifying any malaria drug decisions. ${ }^{[9]}[10]$ Although chloroquine remains a very adequate therapy for vivax malaria in most areas, no one can now depend on chloroquine to cure falciparum malaria. The delayed evolution of chloroquineresistance unfortunately lulled physicians into a false sense of security which must now be dispelled. The anti-inflammatory properties of chloroquine may make patients with nonspecific symptoms feel better, but chloroquine alone cannot be regarded as adequate treatment for falciparum malaria.

Anti-folate-resistance: Anti-folate drugs block the parasite's synthesis of tetrahydrofolate thus stopping nucleic acid synthesis. Sequential blockades of the synthesis pathway using both dihydrofolate reductase by pyrimethamine and dihydropteroate synthetase by sulfadoxine can often produce a cure. ${ }^{[11]}$ Unfortunately, falciparum parasites are able to quickly accumulate multiple genetic mutations producing enzymes resistant to such drugs. In 1970s, sulfadoxine/pyrimethamine (SP) failed as primary therapy in a very short period of time once it was in general use in Thailand. These same resistance genes have rapidly spread across Asia and into Africa such that treatment with a single administration of sulfadoxine/pyrimethamine often fails to cure uncomplicated falciparum infections. ${ }^{[1]}$ Once a drug fails to cure a substantial number of infections, its ability to be used in combination with another more effective drug, is also severely limited.

Mefloquine-resistance: In retrospect, it is possible to see that mefloquine-resistant parasites pre-existed the introduction of mefloquine. In practical terms this meant that the widespread use of mefloquine allowed the selection of multiple drug-resistant falciparum parasites. Despite the operational desirability of giving treatment in a single dose, such long-acting drugs such as mefloquine or sulfadoxine/pyrimethamine hold within themselves the root of their own demise. The long elimination half-life of mefloquine allows parasites to encounter sub-inhibitory concentrations in persons treated weeks or months previously. Thus the selection and establishment of mefloquine resistance is only a matter of time once it is widely used alone without combination with another effective drug. ${ }^{[12]}$

Atovaquone-resistance: Atovaquone is a relatively new antimalarial drug that blocks the parasite's cytochrome electron transfer system. Unfortunately, a single nucleotide mutation in the cytochrome B gene engenders very high grade drug-resistance even in a single patient. Atovaquone cannot be used alone; indeed it is only available in a combination tablet with Proguanil. Atovaquone/Proguanil combination given as an oral dose over a period of three days resulted in a very high cure rate in uncomplicated falciparum patients. Atovaquone's long half-life predisposes it to selection of drug resistant strains over time. Considering the expensive nature of the drug, its widespread use is unlikely thus delaying the inevitable drug-resistance to Atovaquone. ${ }^{[13]}$

Multiple-drug resistance: Multiple drug- resistance occurs when a parasite that already is resistant to one class of anti-malarial drugs develops resistance to another separate class of drug. ${ }^{[14]}$ Combination chemotherapy in malaria is typically formed when a rapidly acting drug (quinine or artemisinin) is combined with a slower acting drug (tetracycline, mefloquine etc.) given over enough time (at least four parasite generations which is about eight days) to kill any residual parasites. ${ }^{[15]}$ Currently available combinations which have been proven effective in field trials include quinine-tetracycline, chlorproguanil-dapsone, artemetherlumefantrine and the previously mentioned atovaquone-proguanil. As with any medication, each combination has its own advantages and disadvantages based on expensiveness, tolerability and ease of administration. ${ }^{[16][17]}$

\section{Artemisinin and its derivatives}

Malaria has been known since time immemorial, but it was centuries before the true causes were understood. Previously, it was thought that "maimsa" (bad air or gas from swamps"malairia") caused the disease. Surprisingly in the view of this, some ancient treatments were remarkably effective. An infusion of qinghao (Artemisia апnиa) has been used for at least the last 2000 years in China, its active ingredient (artemisinin) having only recently been scientifically identified. Irrespective of the practical problems of quinine, particularly low patient compliance from frequent adverse events such as tinnitus, artemisinin compounds are increasingly used for falciparum treatment. Derived from the Chinese pharmacopoeia and a type of wormwood plant, artemisinin compounds exist in a variety of derivatives most of which appear to have as their active metabolite dihydro artemisinin. The major advantages of artemisinin compounds are their fast action in clearing the blood of parasites and preventing the emergence of the gametocyte transmission stages. ${ }^{[14]}{ }^{[15]}$ Both of these characteristics minimize the development of drugresistance and encourage further use of artemisinin compounds. Artemisinin and structurally related compounds act via mechanisms that are distinct from other anti-malarial classes, including those that inhibit well defined targets such as enzymes of folate biosynthesis, the DOXP reductase pathway or the cytochrome electron transport system. The peroxide within the 1 , 2, 4-trioxane system of artemisinin is essential for anti-malarial activity. Thus, artemisinin compounds lacking a peroxide oxygen atom such as the desoxy compounds are devoid of activity. Hence, the peroxide structure has therefore, become a focus for considerable chemical analysis aimed at trying to understand how artemisinins work. ${ }^{[16]}$ In turning to 'activation' of artemisinin by haem, it was noted that from the early trophozoite stage, the malaria parasite catabolizes hemoglobin as a source of amino acids 


\section{Maqbool et al, Malaria and its treatment approaches}

in an acidic, oxygen-rich lysosome like digestive vacuole. ${ }^{[17]}$ The catabolism releases ferriprotoporphyrin IX (haem), and the haem undergoes a non-enzymatic dimerization to form haemozoin, the insoluble, highly crystalline malaria pigment. Virtually all of the haem is converted into haemozoin, and very little is degraded by other pathways. ${ }^{[18]}$ Of the total iron present in Plasmodium falciparum trophozoites, approximately $92 \%$ is located within the food vacuole, and of this, approximately $88 \%$ is in the form of haemozoin. Indeed, haemozoin is the only detectable iron species in trophozoites, and the haemozoin loading in the food vacuole is very high. The progenitor of haemozoin, namely haem, has been studied as an 'activator' of the artemisinins. [14C]-Artemisinin was found to interact with haem, either within the parasite, or in vitro in a medium containing ferriprotoporphyrin IX chloride at $\mathrm{pH}$ 7.5- 7.8. Artemether is a potent and rapidly acting anti-malarial agent which is used as first-line therapy for the treatment of severe multi-drug resistant malaria. It is effective against $P$. vivax as well as chloroquine-sensitive and chloroquine-resistant strains of $P$. falciparum. Although artemether has good therapeutic potential in malaria, its poor aqueous solubility is a major limiting factor that hampers its clinical efficiency. ${ }^{[19]}{ }^{[20]}$ Currently, artemether is available as capsules containing $40 \mathrm{mg}$ and tablets containing 50 $\mathrm{mg}$ for oral therapy and as injectable solution vials for deep Intramuscular treatment of $80 \mathrm{mg} / \mathrm{ml}$ and $20 \mathrm{mg} / \mathrm{ml}$ (for children) for severe malaria infections. Currently available oily IM injection of artemether causes considerable pain to patients on injection and it also shows slow and erratic absorption of artemether on IM administration. Thus, development of parenteral formulation of artemether to achieve effective and optimal utilization of artemether is needed for quick eradication of the malarial infection. It has been demonstrated that IV delivery of artemether results in highest availability to body as compared to all other routes such as intramuscular or intraperitoneal and can lead to quick eradication of the infection. However, the oily nature of current parenteral formulation of artemether makes it unsuitable for administration by intravenous (IV) route. Researchers have explored the potential of other novel delivery approaches such as microemulsions, liposomes ${ }^{[21]}$, and nanostructured lipid carriers ${ }^{[22]}$ to enable IV delivery of artemether and also improve the therapeutic efficacy of artemether.

\section{CONCLUSION}

The emergence of resistance to the conventional anti-malarial drugs is a worrying phenomenon with respect to malaria; it is such a widespread and deadly disease, that the consequences of failed treatment are very high. Resistance can be caused by many factors, at the level of the drug, the human host, the mosquito host and also the malaria parasite itself. With successive generations, natural selection will lead to the evolution of strains of malaria parasites which are firmly resistant to that drug. It is crucially important for people to be given accurate doses of medication, to ensure that they complete the full course of treatment, and that once treatment has been completed; they are accurately tested as negative for the malaria parasite. Finally, there are factors related to the affinity of the malaria parasite to its vector mosquito hosts which can lead to the emergence of drug resistant strains. It is for these reasons that malaria treatment and control programmes are now being very careful with the ways in which they administer drugs and monitor infections, in order to limit any further resistance developing; similarly, pharmaceutical and biochemical researchers are constantly on the look-out for new compounds or methods of killing malaria parasites, which can be developed into new forms of treatment.

\section{ACKNOWLEDGEMENT}

Authors are extremely thankful to the referred authors for their incredible findings that helped us in compiling this exhausted review article.

\section{CONFLICT OF INTEREST}

The author declares that they have no competing interests.

\section{REFERENCE}

1. Gaur R, Cheema HS, Kumar Y, Singh SP, Yadav DK, Darokar MP, Khan F, Bhakuni RS. In vitro antimalarial activity and molecular modeling studies of novel artemisinin derivatives. RSC Advances. 2015; 59:26822689.

2. Butcher GA. Antimalarial drugs and the mosquito transmission of Plasmodium. International Journal of Parasitology. 1997; 27(9):975-987.

3. Shanks GD. Treatment of falciparum malaria in the age of drug resistance. Journal of Post Graduate Medicine. 2006; 52(4):277-280.

4. Hastings I. How artemisinin-containing combination therapies slow the spread of antimalarial drug resistance. Trends in Parasitology. 2011; 27(2): 67-72.

5. White NJ. Antimalarial drug-resistance. Journal of Clinical Investigations. 2004; 113: 1084-1092.

6. White NJ, Pongtavornpinyo W. The de novo selection of drug-resistant malaria parasites. ProceedingsBiological Sciences. 2003; 270(1514): 545-554.

7. Wongsrichanalai C, Pickard AL, Wernsdorfer WH, Meshnick SR. Epidemiology of drug-resistant malaria. The Lancet- Infectious Diseases. 2002; 2(4): 209-218.

8. Dutta P, Mahanta J. Response of Plasmodium Falciparum to chloroquine in Digboi area of Assam, India. The Journal of Communicable Diseases. 1996; 28(3): 212-214.

9. Campbell CC, Chin W, Collins WE, Teutsch SM, Moss DM. Chloroquine-resistant Plasmodium falciparum from East Africa: cultivation and drug sensitivity of the Tanzanian I/CDC strain from an American tourist. Lancet. 1979; 2(8153):1151-1154.

10. Peterson DS, Walliker D, Wellems TE. Evidence that a point mutation in dihydrofolate reductase-thymidylate synthase confers resistance to pyrimethamine in falciparum malaria. Proceedings of National Academy of Science of USA. 1988; 85(23): 9114-9118.

11. Kuile FO, Luxemburger C, Nosten F, Thwai KL, Chongsuphajaisiddhi T, White NJ. Predictors of Mefloquine treatment failure: A prospective study of 1590 patients with uncomplicated falciparum malaria. Transactions of the Royal Society of Tropical Medicine and Hygiene. 1995; 89(6): 660-664. 


\section{Maqbool et al, Malaria and its treatment approaches}

12. McKeage K, Scott L. Atovaquone/Proguanil: A review of its use for the prophylaxis of Plasmodium falciparum malaria. Drugs. 2003; 63(6): 597-623.

13. Price RN, Nosten F, Luxemburger C, Kham A, Brockman A, Chongsuphajaisiddhi T, White NJ. Artesunate versus artemether in combination with mefloquine for the treatment of multidrug-resistant falciparum malaria. Transactions of the Royal Society of Tropical Medicine and Hygiene. 1995; 89(6): 523527.

14. Price RN, Nosten F, Luxemburger C, van Vugt M, Phaipun L, Chongsuphajaisiddhi T, White NJ. Artesunate/Mefloquine treatment of multi-drug resistant falciparum malaria. Transactions of the Royal Society of Tropical Medicine and Hygiene. 1997; 91(5): 574577.

15. Krishna S, Uhlemann AC, Haynes RK. Artemisinins: mechanisms of action and potential for resistance; Drug Resistance updates- reviews and commentaries in antimicrobial and anticancer chemotherapy. 2004; 7(45): 233-244.

16. Sullivan DJ. Theories on malarial pigment formation and quinolone action. International Journal of Parasitology. 2002; 32(13): 1645-1653.

17. Egan TJ, Combrinck JM, Egan J, Hearne GR, Marques HM, Ntenteni S, Sewell BT, Smith PJ, Taylor D, van Schalkwyk DA, Walden JC. Fate of haem iron in the malaria parasite Plasmodium falciparum. The Biochemical Journal. 2002; 365(Pt 2): 343-347.

18. Tayade NG, Nagarsenkar MS. Development and evaluation of artemether parenteral microemulsion. Indian Journal of Pharmaceutical Sciences. 2010; 72(5): 637-640.

19. Karbwang J, Na-Bangchang K, Congpuong K, Molunto $\mathrm{P}$, Thanavibul A. Pharmacokinetics and bioavailability of oral and intramuscular Artemether. European Journal of Clinical Pharmacology. 1997; 52(4): 307-310.

20. Chimanuka B, Gabriels M, Detaevernier MR, PlaizierVercammen JA. Preparation of $\beta$-Artemether liposomes, their HPLC-UV evaluation and relevance for clearing recrudescent parasitemia in P. chabaudi malaria infected mice. Journal of Pharmaceutical and Biomedical Analysis. 2002; 28(1): 13-22.

21. Joshi M, Pathak S, Sharma S, Patravale V; Design and in vivo pharmacodynamics evaluation of nanostructured lipid carriers for parenteral delivery of Artemether: Nanoject; International Journal of Pharmaceutics. 2008; 364(1): 119-126.

22. Chiou WL, Riegelman S. Pharmaceutical applications of solid dispersion systems. Journal of Pharmaceutical Sciences. 1971; 60(9): 1281-1302. 